\title{
Ginkgolide B-mediated therapeutic effects on perioperative neurocognitive dysfunction are associated with the inhibition of iNOS-mediated production of NO
}

\author{
TING LUO $^{1 *}$, DANDAN LIN ${ }^{1 *}$, YANAN HAO $^{1}$, RONG SHI $^{1}$, CHANGWEI WEI $^{1}$, \\ WENZHEN SHEN ${ }^{1}$, ANSHI WU ${ }^{1}$ and PEILI HUANG ${ }^{2,3}$ \\ ${ }^{1}$ Department of Anesthesiology, Beijing Chao-Yang Hospital, Capital Medical University, Beijing 100020; \\ ${ }^{2}$ School of Public Health; ${ }^{3}$ Beijing Key Laboratory of Environmental Toxicology, \\ Capital Medical University, Beijing 100069, P.R. China
}

Received March 26, 2020; Accepted March 17, 2021

DOI: $10.3892 / \mathrm{mmr} .2021 .12176$

\begin{abstract}
Perioperative neurocognitive dysfunction (PND) is a prevalent neurological complication after anesthesia and surgery. Ginkgolide B (GB) has been suggested to improve lipopolysaccharide-induced learning and memory impairment. The present study aimed to investigate whether GB serves a protective role against PND by inhibiting inducible nitric oxide synthase (iNOS) and nitric oxide (NO). Abdominal surgery was performed on 10- to 12-week-old male C57BL/6 mice under isoflurane anesthesia. Prior to surgery, $1400 \mathrm{~W}$ (a specific iNOS inhibitor) and GB were administered via intraperitoneal injection. Open field and fear conditioning tests were conducted to assess cognitive function on postoperative days 1 and 3. Biochemical assays were performed to evaluate alterations in NO, malondialdehyde (MDA) and superoxide dismutase (SOD) levels. Western blotting was performed to measure iNOS expression in the hippocampus on postoperative day 1 . In addition, hematoxylin and eosin staining was performed to detect the neuronal morphology in the hippocampus. Following treatment with $1400 \mathrm{~W}$ or GB, surgery-induced cognitive dysfunction was improved. Compared with the control group, the surgery group exhibited significant overproduction of iNOS and MDA in the hippocampus on postoperative day 1. Higher levels of NO were also detected in the hippocampus and prefrontal cortex
\end{abstract}

Correspondence to: Dr Anshi Wu, Department of Anesthesiology, Beijing Chao-Yang Hospital, Capital Medical University, 8 Gongtinan Road, Beijing 100020, P.R. China

E-mail:wuanshi@hotmail.com

Professor Peili Huang, School of Public Health, Capital Medical University, 10 Xitoutiao, You An Men, Beijing 100069, P.R. China E-mail: huangpl@ccmu.edu.cn

*Contributed equally

Key words: ginkgolide B, perioperative neurocognitive dysfunction, inducible nitric oxide synthase, nitric oxide, apoptosis of the surgery group on postoperative day 1. Furthermore, pretreatment with $1400 \mathrm{~W}$ or GB significantly inhibited the surgery-induced elevation of NO and MDA in brain tissues. Moreover, GB pretreatment significantly inhibited surgery-induced downregulation of SOD and upregulation of iNOS. Surgery-induced increases in neuronal loss and the $\mathrm{Bax} / \mathrm{Bcl}-2$ ratio in the hippocampus were significantly inhibited by pretreatment with GB. Collectively, the results of the present study demonstrated that the therapeutic effects of GB on PND were associated with inhibition of iNOS-induced NO production, increased SOD, and the alleviation of neuronal loss and apoptosis.

\section{Introduction}

Perioperative neurocognitive disorder (PND) is a common cognitive complication of surgery, especially among older patients, which is characterized by progressive cognitive deterioration in the preoperative or postoperative period. PND has several clinical manifestations, including attention deficits, learning disabilities and memory loss $(1,2)$. In the long term, PND can result in prolonged hospitalization, decreased quality of life and increased financial burden on the family and society (3). Nevertheless, the mechanisms underlying PND are not completely understood. Netto et al (4) reported that PND model rats displayed increased levels of nitrite/nitrate, which are metabolites of nitric oxide (NO). Zhang et al (5) demonstrated that anesthesia and surgery increased reactive oxygen species (ROS) and decreased the level of antioxidant enzymes in the hippocampus of mice. The superoxide anion $\left(\mathrm{O}_{2}{ }^{-}\right)$is an important specie of ROS (6).

Inducible NO synthase (iNOS), which is primarily expressed in pathological conditions, is able to produce large amounts of NO. Appropriate levels of NO can serve a neuroprotective role, but large quantities of NO are neurotoxic (7). A high level of NO has been indicated to serve as a major mediator in neurodegenerative diseases $(8,9)$. It was reported that prolonged NO exposure could result in overproduction of cytoplasmic $\tau$ protein in SH-SY5Y cells (10). NO also serves an important role in the process of Parkinson's disease by targeting the 
mitochondria, leading to inhibition of the respiratory chain (11). A higher concentration of $\mathrm{NO}$ reacts with $\mathrm{O}_{2}{ }^{--}$to generate a large amount of peroxynitrite $\left(\mathrm{ONOO}^{-}\right)$. $\mathrm{ONOO}^{-}$is a highly cytotoxic superoxide that can lead to neuronal damage by oxidizing lipids, proteins and DNA (12). However, the roles of iNOS and NO in the process of PND are not completely understood.

Ginkgolide B (GB) is one of the terpene lactone components extracted from Gingko biloba (13) It has been reported that GB protects SH-SY5Y cells against A $\beta$ toxicity (14). GB can also inhibit oxidative stress to protect neurons from cerebral ischemia injury (15). GB and its analog XQ-1H have been reported to exert neuroprotective effects by downregulating iNOS and NO in cerebral ischemia and reperfusion (16-18). However, to the best of our knowledge, no previous studies have investigated GB as a protective treatment for PND.

The present study hypothesized that iNOS was involved in the process of PND by promoting the production of $\mathrm{NO}$, and the neuroprotective effect of GB on PND was associated with the inhibition of iNOS-mediated production of NO. Therefore, the present study used the specific iNOS inhibitor $1400 \mathrm{~W}$ to investigate the role of iNOS-mediated production of NO in the process of PND. The effects of GB on PND, iNOS-mediated production of $\mathrm{NO}$, neuronal apoptosis and morphological alterations of neurons in the hippocampus were also investigated. The results of the present study may suggest a novel effective pretreatment for PND.

\section{Materials and methods}

Animals. Male C57B6/L mice (age, 10-12 weeks; weight, 23-28 g; n=96) were purchased from Beijing Vital River Laboratory Animal Technology Co., Ltd.. Mice were housed under controlled conditions with 12-h light/dark cycles and were provided with a standard diet and water ad libitum. The temperature was controlled at $26 \pm 1^{\circ} \mathrm{C}$ and the humidity was maintained at $50 \pm 10 \%$. All experiments were approved by the Animal Experiments and Experimental Animal Welfare Committee of Capital Medical University (ethical review number: 2018-0003; Beijing, China) and performed under the regulations of the Medical Research Center of Beijing Chao-Yang Hospital (Beijing, China).

Intraperitoneal administration of $1400 \mathrm{~W}$ and GB. For the intervention study, $1400 \mathrm{~W}$ and GB were intraperitoneally administered before surgery. To determine the effective dosage, three male C57B6/L mice (age, 10-12 weeks; weight, 23-28 g) of each group received different doses of 1400W (10 and $20 \mathrm{mg} / \mathrm{kg}$ ) and GB (10 and $20 \mathrm{mg} / \mathrm{kg}$ ) in the preliminary experiment. These mice were housed under the same conditions as aforementioned. The results demonstrated that the administration of $20 \mathrm{mg} / \mathrm{kg} 1400 \mathrm{~W}$ at $30 \mathrm{~min}$ prior to surgery prevented surgery-induced cognitive impairment. For pretreatment with $\mathrm{GB}$, repeated administration of GB (20 mg/ $\mathrm{kg} /$ day) for 5 days prior to surgery improved exploratory laparotomy-induced cognitive dysfunction (data not shown). Subsequently, mice were randomly divided into the following groups: i) Control $(n=32$, mice received no treatment); ii) surgery $(n=32)$; iii) surgery + GB [mice received GB (20 mg/kg/day) for 5 days prior to surgery, $\mathrm{n}=16$ ]; and iv) surgery $+1400 \mathrm{~W}$ [mice received $1400 \mathrm{~W}(20 \mathrm{mg} / \mathrm{kg})$ at $30 \mathrm{~min}$ prior to surgery, $\mathrm{n}=16]$.
Abdominal surgery. The exploratory laparotomy was performed as previously described $(19,20)$. The mice were anesthetized with $3.0 \%$ isoflurane for induction and $1.5 \%$ isoflurane for maintenance. A $1.5-\mathrm{cm}$ vertical incision was made in the midline of the abdomen. A sterile metal probe was inserted into the abdominal cavity and the abdominal viscera was explored. The entire procedure lasted $\sim 10 \mathrm{~min}$. Subsequently, the peritoneum, abdominal muscles and skin were sutured layer-by-layer using 4-0 silk thread sutures. The mice were placed into a chamber for recovery from anesthesia. Following recovery, the mice were placed back into their cages with ad libitum access to water and food.

Open field test (OFT). The OFT was performed as previously described (21). The different groups of mice were subjected to the OFT on postoperative days 1 and $3(n=8)$. Each mouse was gently placed in the center of the Plexiglas ${ }^{\circledR}$ open field apparatus $\left(40 \mathrm{~cm}^{3}\right)$ for $5 \mathrm{~min}$. Movement was monitored and recorded using a video tracking system. The total moving distance in the chamber and duration in the central area were recorded to assess locomotor activity and exploration (22).

Fear conditioning test (FCT). The FCT was performed as previously described, with minor adjustments $(n=8$ mice/group) $(23,24)$. All mice were trained for fear conditioning $2 \mathrm{~h}$ prior to surgery. For fear conditioning, each mouse was placed into a conditioning chamber, and a video and software (Xeye FCS; Beijing MacroAmbiton S\&T Development Co., Ltd.) were used to record and analyze the movement of the mice. The mice were allowed to explore for $180 \mathrm{sec}$ before exposure to both tone and foot shock (tone: $2,500 \mathrm{~Hz}, 80 \mathrm{Db}, 30 \mathrm{sec}$; foot shock: $0.7 \mathrm{~mA}, 2 \mathrm{sec}$ ), three times at 60 -sec intervals. Subsequently, the mouse was removed from the chamber and returned to its cage. In addition, $70 \%$ alcohol was used to wipe the grid floor during the interval between testing of different animals.

The mice underwent the contextual and cued tests on postoperative days 1 and 3 . For the contextual test, each mouse was placed into the same chamber for $360 \mathrm{sec}$ without tone or foot shock. For the cued test, each mouse was placed into the same chamber for $180 \mathrm{sec}$ with tone but without foot shock. There was a 2-h interval between the two tests. Cognitive function was determined as the amount of time that freezing behavior was recorded by the video tracking system. The contextual test reflected hippocampus-dependent memory function and the cued test reflected hippocampus-independent memory function (25).

Biochemical analysis. On postoperative day 1 and 3, mice were transcardially perfused with ice-cold PBS under anesthesia (3.0\% isoflurane for induction and $1.5 \%$ isoflurane for maintenance). Subsequently, the mice were euthanized via cervical dislocation, and brain tissue was harvested and immediately stored at $-80^{\circ} \mathrm{C}$. The cortex and hippocampus were homogenized in PBS. Following centrifugation of the homogenate at $1,431 \mathrm{x} \mathrm{g}$ for $10 \mathrm{~min}$ at $4^{\circ} \mathrm{C}$, the supernatant was collected for biochemical analysis. The total protein concentration of the supernatant was determined using the bicinchoninic acid protein assay (cat. no. P0012S; Beyotime Institute of Biotechnology). NO, malondialdehyde (MDA) and superoxide dismutase (SOD) levels in the cortex and hippocampus were measured using NO assay kit (nitrate reductase method; cat. no. A012-1-2; Nanjing Jiancheng Bioengineering Institute), 

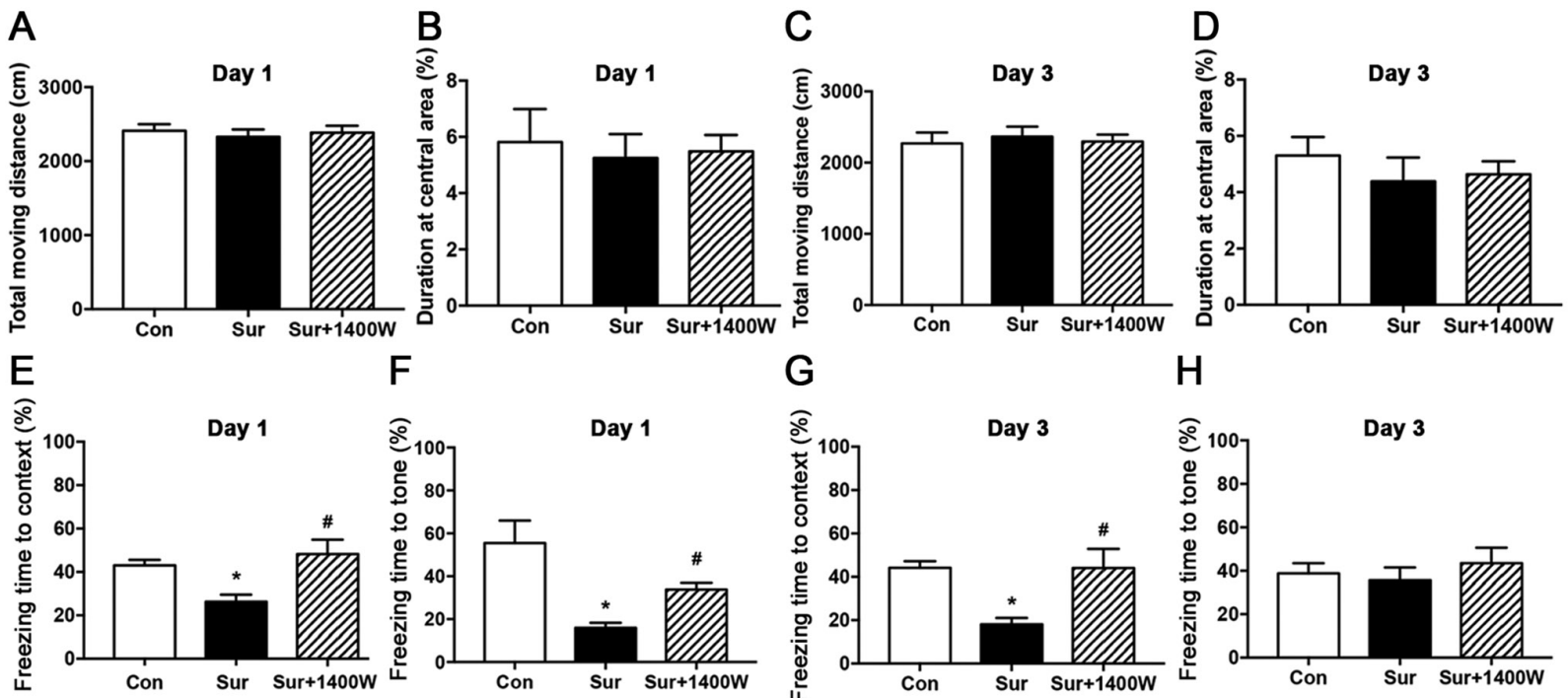

Figure 1. Effect of $1400 \mathrm{~W}$ treatment on perioperative neurocognitive dysfunction. (A) Total moving distance and (B) duration at central area in the OFT at postoperative day 1. (C) Total moving distance and (D) duration at the central area in the OFT at postoperative day 3. Freezing time in the (E) contextual and $(\mathrm{F})$ cued tests at postoperative day 1. Freezing time in the $(\mathrm{G})$ contextual and $(\mathrm{H})$ cued tests at postoperative day 3. Compared with the control group, surgery and $1400 \mathrm{~W}$ did not significantly affect the exploratory and locomotor activities as demonstrated by the OFT results. 1400W significantly inhibited surgery-induced hipppocampus-dependent cognitive impairment on postoperative days 1 and 3. Hippocampus-independent cognitive impairement on postoperative day 1 was also improved by $1400 \mathrm{~W}$. Data are presented as the mean $\pm \mathrm{SEM}$ ( $\mathrm{n}=8$ per group). ${ }^{*} \mathrm{P}<0.05$ vs. control; ${ }^{*} \mathrm{P}<0.05$ vs. surgery. OFT, open field test; Con, control; Sur, surgery.

MDA assay kit (TBA method; cat. no. A003-1-2; Nanjing Jiancheng Bioengineering Institute), total SOD assay kit (hydroxylamine method; cat. no. A001-1-2; Nanjing Jiancheng Bioengineering Institute) according to the manufacturer's protocols. Biochemical analyses were performed at $4^{\circ} \mathrm{C} .(\mathrm{n}=6)$.

Western blotting. Total protein was extracted from hippocampal tissues using Minute ${ }^{\mathrm{TM}}$ Total Protein Extraction kit (cat. SD-001/SN-002, Invent Biotechnologies, Inc.) with a protease and phosphatase inhibitor cocktail (Thermo Fisher Scientific, Inc.). The homogenate was centrifuged at 13,147 x g for $2 \mathrm{~min}$ at $4^{\circ} \mathrm{C}$. The supernatant was obtained and the protein concentrations in the supernatant were quantified using a BCA Protein assay reagent kit (Beyotime Institute of Biotechnology). Proteins (30 $\mu \mathrm{g}$ per lane) were separated on a $10 \%$ gel using SDS-PAGE and transferred onto PVDF membranes. Following blocking with $5 \%$ skimmed milk for $1 \mathrm{~h}$ at $37^{\circ} \mathrm{C}$, the membranes were incubated overnight at $4^{\circ} \mathrm{C}$ with primary antibodies in 5\% milk targeted against iNOS, ( $\mathrm{n}=6$ mice/group) $(1: 1,000$; cat no. ab178945; Abcam), Bax, (n=4), (1:1,000; cat. no. ab32503; Abcam), Bcl-2, (n=4) (1:2,000; cat. no. ab182858; Abcam) and $\beta$-actin (1:1,000; cat. no. ab8226; Abcam). After washing three times with TBS- $0.05 \%$ Tween-20, the membranes were incubated with secondary antibodies (1:5,000; cat. nos. 31464 and 31450; Thermo Fisher Scientific, Inc.) in TBS for $30 \mathrm{~min}$ at $37^{\circ} \mathrm{C}$. Subsequently, the membranes were incubated with chemiluminescent HRP substrate (Thermo Fisher Scientific, Inc.). Protein bands were visualized using the ChemiDoc XRS system (Version 3.0; Bio-Rad Laboratories, Inc.). Protein expression was semi-quantified using Image Lab software (Bio-Rad Laboratories, Inc.) with $\beta$-actin as the loading control.

Hematoxylin and eosin staining. Mice were perfused with ice-cold PBS and sacrificed by cervical dislocation. The brain tissue was immediately collected and fixed with $4 \%$ paraformaldehyde for $24 \mathrm{~h}$ at room temperature. After dehydration with alcohol (30-100\%), the brain tissue was embedded in paraffin. Subsequently, 4- $\mu \mathrm{m}$ thick coronal sections were prepared. The brain sections were dewaxed with xylene, followed by hydrating with gradient alcohol series (100-70\%). The brain sections were then placed in $0.5 \%$ hydrochloric acid for $10 \mathrm{sec}$ and in water for $10 \mathrm{~min}$. The slices were later stained with hematoxylin for $5 \mathrm{~min}$ and counterstained with eosin for 3 min. An optical microscope (Olympus Corporation) was used to observe the slices, $(n=3)$.

Statistical analysis. All experiments were performed once; the number of mice in each group varied between three and eight. Data are presented as the mean \pm SEM. Statistical analyses were performed using GraphPad Prism V7 (GraphPad Software, Inc.). Comparisons among multiple groups were analyzed using one-way ANOVA followed by Tukey's post hoc test. $\mathrm{P}<0.05$ was considered to indicate a statistically significant difference.

\section{Results}

$1400 \mathrm{~W}$ pretreatment improves abdominal surgery-induced learning and memory impairments. The OFT was performed to evaluate locomotor activity and exploration of the mice prior to the FCT by recording the total moving distance and duration at the central area. No significant differences in the OFT results were identified among the three groups on postoperative days 1 and 3, suggesting that locomotor activity and exploration were not affected by surgery or pretreatment with $1400 \mathrm{~W}$ (P $>0.05$; Fig. 1A-D).

Subsequently, the FCT was conducted to evaluate learning and memory capacities. The freezing time to context in the 

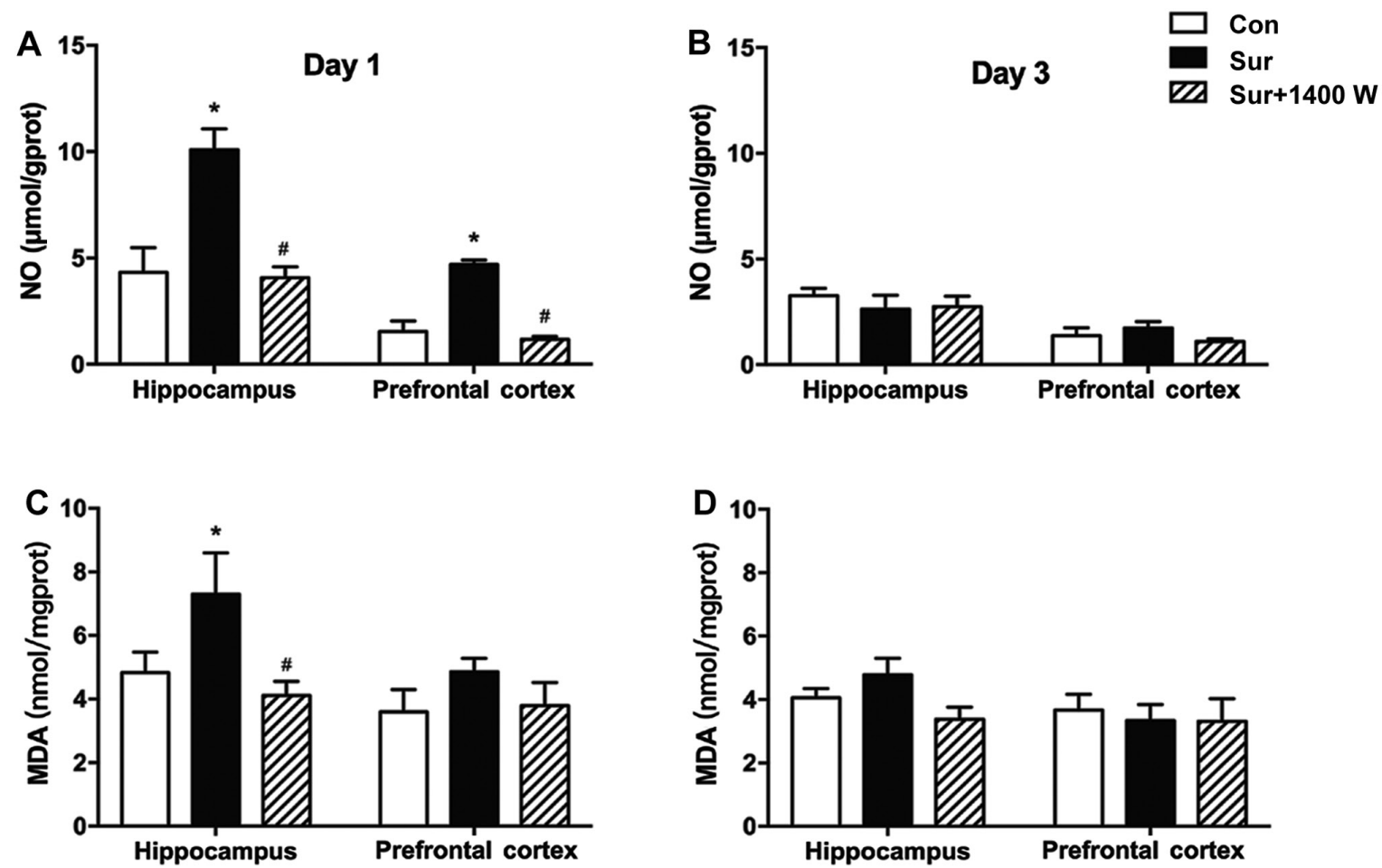

Figure 2. Effect of $1400 \mathrm{~W}$ treatment on NO and MDA. (A) 1400W administration significantly prevented surgery-induced increases in NO levels in the hippocampus and prefrontal cortex on postoperative day 1. (B) No significant difference in NO levels in the brain tissue was observed among the three groups on postoperative day 3. (C) Surgery-induced MDA overproduction in the hippocampus on postoperative day 1 was significantly inhibited by $1400 \mathrm{~W}$. (D) No significant differences in MDA levels in the brain tissue were observed among the three groups on postoperative day 3. Data are presented as the mean \pm SEM ( $\mathrm{n}=6$ per group). ${ }^{*} \mathrm{P}<0.05$ vs. control; ${ }^{~} \mathrm{P}<0.05$ vs. surgery. $\mathrm{NO}$, nitric oxide; MDA, malondialdehyde; Con, control; Sur, surgery.

surgery group was significantly lower on postoperative days 1 and 3 compared with that in the control group $(\mathrm{P}<0.05$; Fig. 1E and G). Similarly, the freezing time to tone was significantly lower in the surgery group compared with that in the control group on postoperative day $1(\mathrm{P}<0.05$; Fig. $1 \mathrm{~F})$. No significant difference in the freezing time to tone among the three groups was observed on postoperative day 3 ( $\mathrm{P}>0.05$; Fig. 1H). Furthermore, compared with the surgery group, the surgery $+1400 \mathrm{~W}$ group displayed significantly higher freezing times in both the contextual and cued tests on postoperative day $1(\mathrm{P}<0.05$; Fig. $1 \mathrm{E}$ and $\mathrm{F})$, and in the contextual test on postoperative day 3 ( $\mathrm{P}<0.05$; Fig. $1 \mathrm{G})$. The results suggested that $1400 \mathrm{~W}$ improved surgery-induced cognitive deficits.

iNOS inhibitor $1400 \mathrm{~W}$ pretreatment decreases NO and MDA levels. Subsequently, whether surgery-induced learning and memory function deficits were associated with iNOS-mediated NO production in the hippocampus was investigated. NO levels in the hippocampus and prefrontal cortex of the surgery group were significantly higher compared with the levels in the control group on postoperative day 1 ( $\mathrm{P}<0.05$; Fig. $2 \mathrm{~A})$. In addition, compared with the control group, the surgery group exhibited a significant increase in the level of MDA in the hippocampus on postoperative day 1 ( $\mathrm{P}<0.05$; Fig. $2 \mathrm{C}$ ). No significant differences in NO and MDA levels were observed among the three groups on postoperative day 3 ( $\mathrm{P}>0.05$; Fig. 2B and D). Compared with those in the surgery group, significantly lower levels of NO and MDA were observed in the hippocampus of the surgery $+1400 \mathrm{~W}$ group on postoperative day 1 . In addition, $\mathrm{NO}$ was decreased in the prefrontal cortex in the surgery $+1400 \mathrm{~W}$ group compared with that in the surgery group $(\mathrm{P}<0.05$; Fig. $2 \mathrm{~A}$ and $\mathrm{C})$.

GB pretreatment attenuates abdominal surgery-induced learning and memory impairments. An OFT was conducted to evaluate alterations in locomotor activity and exploration. No significant differences in the total moving distance and duration at the central area were observed among the three groups ( $\mathrm{P}>0.05$; Fig. 3A-D).

The effects of GB pretreatment on learning and memory capacities were evaluated by performing the FCT. Compared with the control group, the surgery group displayed a significant reduction in freezing time in both the contextual and cued tests on postoperative day $1(\mathrm{P}<0.05$; Fig. $3 \mathrm{E}$ and $\mathrm{F})$. On postoperative day 3 , the freezing time in the contextual test in the surgery group was significantly decreased compared with that of the control group $(\mathrm{P}<0.05$; Fig. $3 \mathrm{G})$. Moreover, abdominal surgery-induced cognitive deficits on postoperative day 1 were significantly reversed by pretreatment with GB $(\mathrm{P}<0.05$; Fig. $3 \mathrm{E}$ and $\mathrm{F})$. In addition, on postoperative day 3, GB pretreatment could increase the freezing time in the contextual test $(\mathrm{P}<0.05$; Fig. $3 \mathrm{G})$, but had no impact on that in the cued test $(\mathrm{P}>0.05$; Fig. $3 \mathrm{H})$.

GB pretreatment inhibits surgery-induced elevations in iNOS, NO and MDA, and alleviates surgery-induced downregulation of SOD. The western blotting results demonstrated that surgery significantly increased the protein expression levels of iNOS in the hippocampus on postoperative day 1 compared with the control group $(\mathrm{P}<0.05$; Fig. 4). GB pretreatment 
A

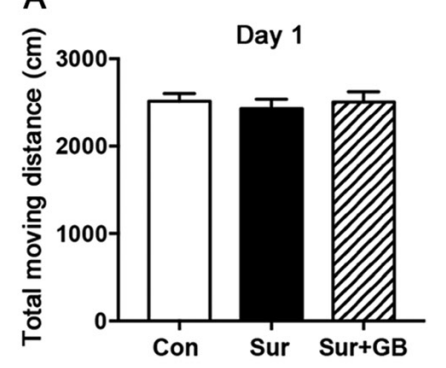

$\mathrm{E}$

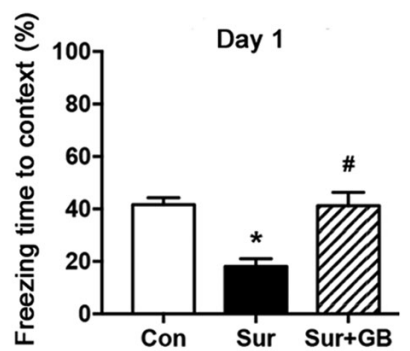

$\mathrm{B}$

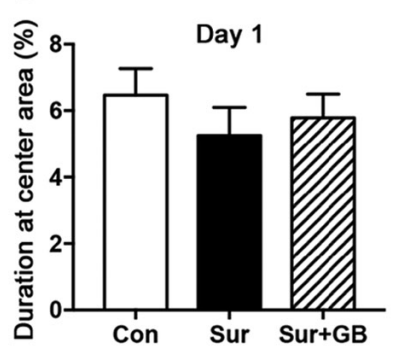

$\mathrm{F}$

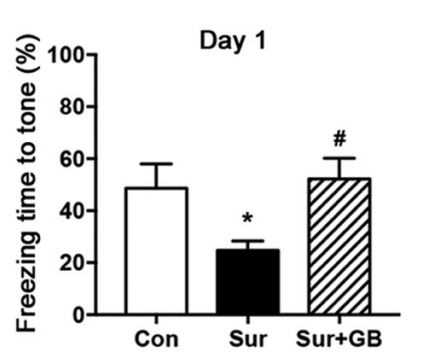

C

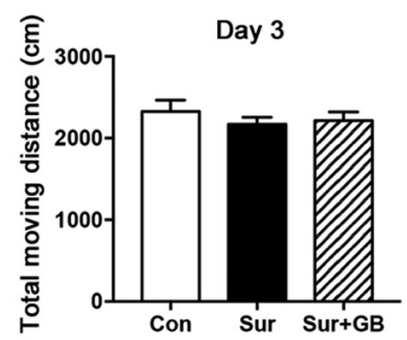

G

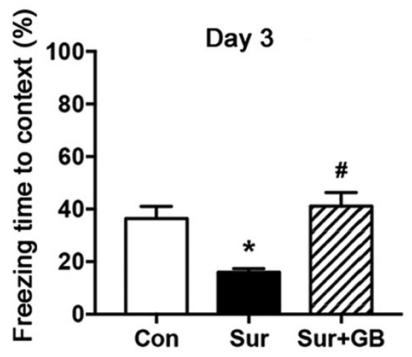

D

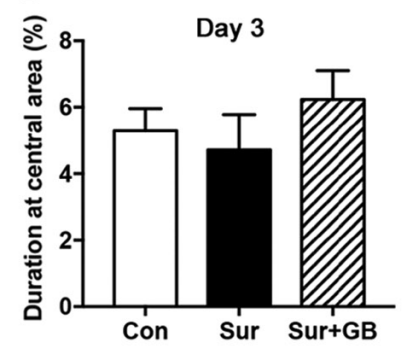

$\mathrm{H}$

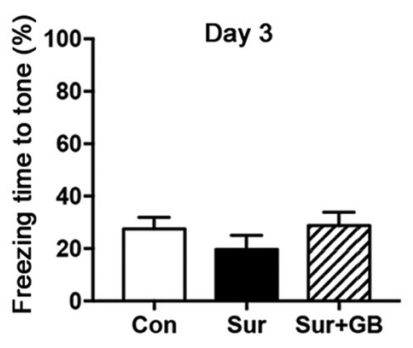

Figure 3. Effect of GB treatment on perioperative neurocognitive dysfunction. (A) Total moving distance and (B) duration at central area in the OFT on postoperative day 1. (C) Total moving distance and (D) duration at central area in the OFT on postoperative day 3. Freezing time during the (E) contextual and (F) cued tests on postoperative day 1. Freezing time during the $(\mathrm{G})$ contextual and $(\mathrm{H})$ cued tests on postoperative day 3 . No significant differences in the OFT results were observed among the three groups. As demonstrated by the fear conditioning test results, GB pretreatment significantly ameliorated surgery-induced hippocampus-dependent cognitive impairment on postoperative days 1 and 3 . Hippocampus-independent cognitive deficit on postoperative day 1 was also alleviated by GB pretreatment. Data are presented as the mean \pm SEM ( $\mathrm{n}=8$ per group). ${ }^{*} \mathrm{P}<0.05$ vs. control; ${ }^{\#} \mathrm{P}<0.05$ vs. surgery. GB, ginkgolide B; OFT, open field test; Con, control; Sur, surgery.
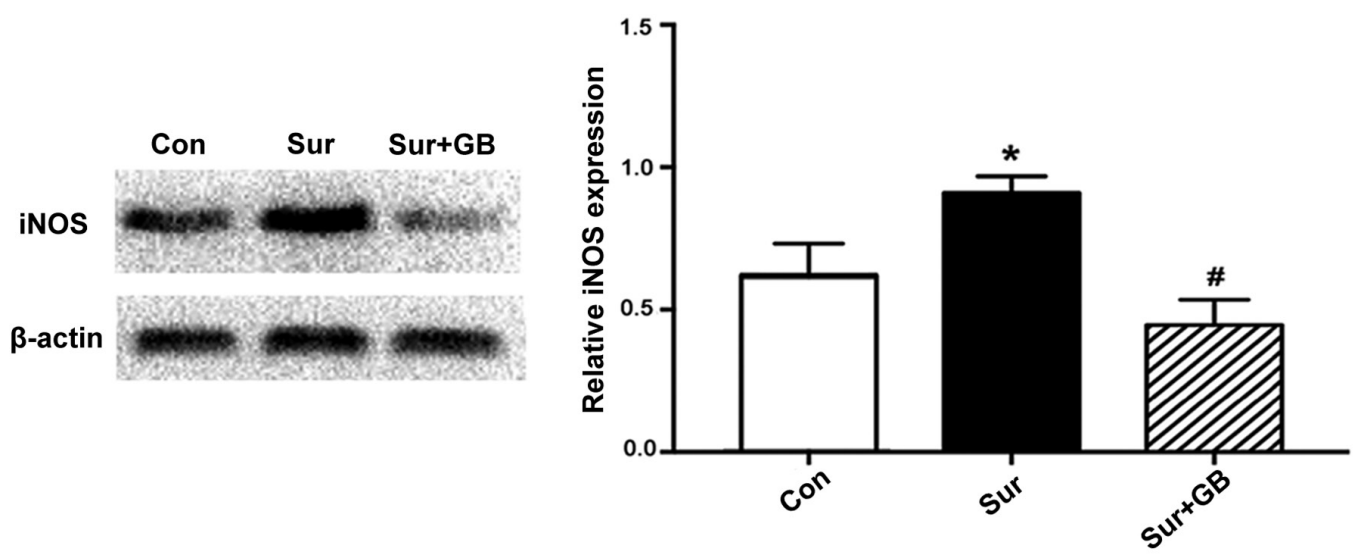

Figure 4. Effect of GB pretreatment on iNOS expression. GB pretreatment significantly inhibited surgery-induced iNOS upregulation in the hippocampus on postoperative day 1 . Data are presented as the mean \pm SEM ( $n=6$ per group). ${ }^{*} \mathrm{P}<0.05$ vs. control; ${ }^{*} \mathrm{P}<0.05$ vs. surgery. GB, ginkgolide $\mathrm{B}$; iNOS, inducible nitric oxide synthase; Con, control; Sur, surgery.

significantly decreased surgery-induced increases in iNOS expression levels ( $\mathrm{P}<0.05$; Fig. 4). Subsequently, the effects of GB pretreatment on the levels of NO, MDA and SOD on postoperative days 1 and 3 were assessed. GB pretreatment significantly alleviated surgery-induced decreases in SOD levels in the hippocampus and prefrontal cortex on postoperative day $1(\mathrm{P}<0.05$; Fig. 5A). GB pretreatment displayed the same effect on surgery-mediated alterations to SOD levels in the hippocampus on postoperative day 3 ( $\mathrm{P}<0.05$; Fig. $5 \mathrm{~B}$ ). GB pretreatment significantly decreased surgery-induced increases in NO and MDA levels in the hippocampus on postoperative day $1(\mathrm{P}<0.05$; Fig. $5 \mathrm{C}$ and $\mathrm{E})$. GB pretreatment also significantly inhibited surgery-induced upregulation of NO levels on postoperative day 1 in the prefrontal cortex $(\mathrm{P}<0.05$;
Fig. 5C). No significant differences were indicated in the levels of NO and MDA among three groups on postoperative day 3 (Fig. 5D and F). The results indicated that GB treatment may alleviate surgery-induced oxidative stress by downregulating iNOS expression.

$G B$ pretreatment decreases the Bax/Bcl-2 ratio and neuronal loss. The neuroprotective role of GB has been reported to be associated with inhibition of the apoptotic protein Bax and elevation of the anti-apoptotic protein Bcl-2 (17). To assess whether surgery altered the $\mathrm{Bax} / \mathrm{Bcl}-2$ ratio in the hippocampus, the protein expression levels of Bax and Bcl-2 were assessed via western blotting. Bcl-2 expression levels were significantly decreased in the surgery group compared with 

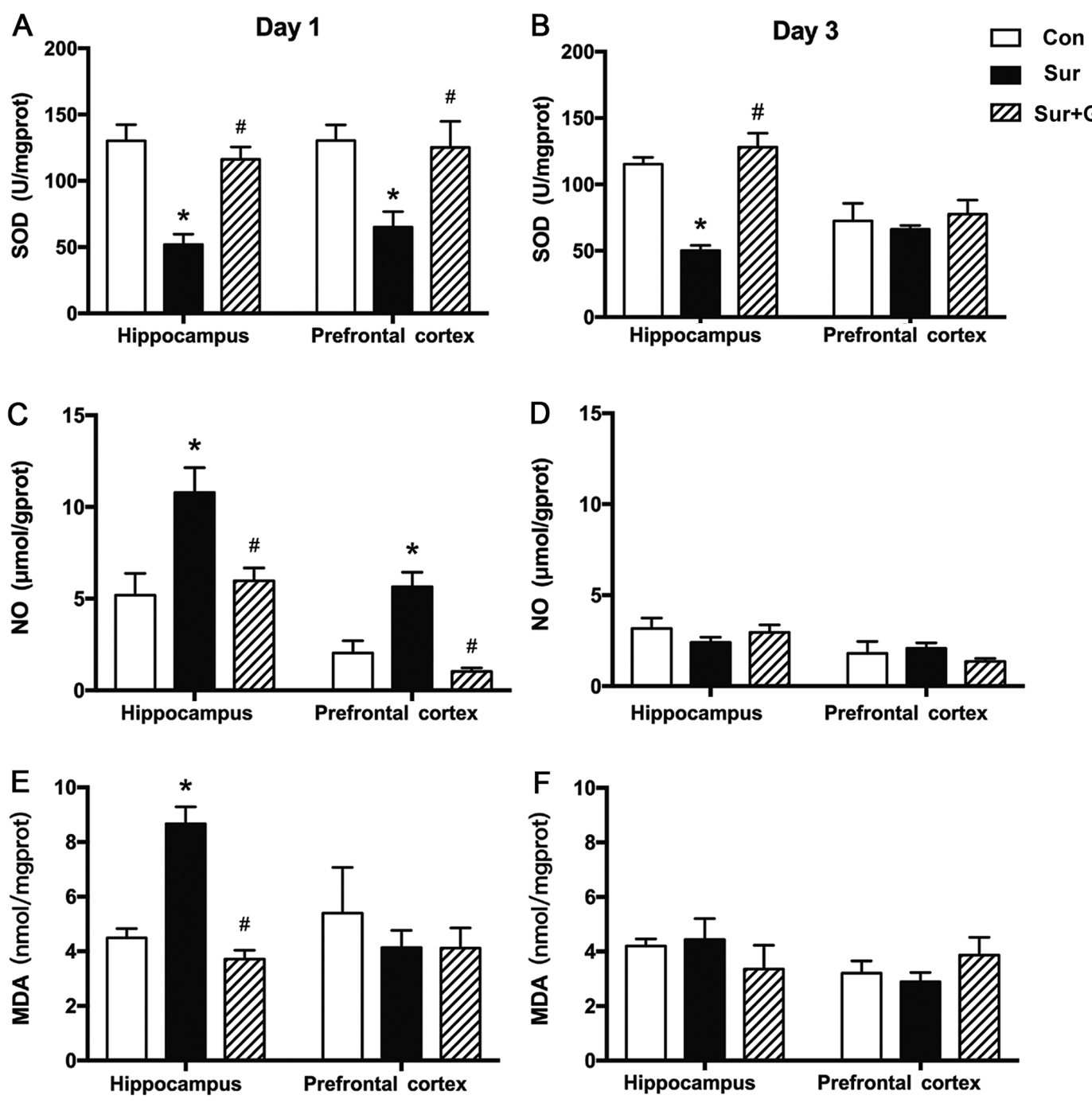

Figure 5. Effect of GB pretreatment on SOD, NO and MDA. GB pretreatment significantly reversed surgery-induced decreases in SOD levels (A) in the hippocampus and prefrontal cortex on postoperative day 1 and (B) in the hippocampus on postoperative day 3. (C) Compared with the control group, surgery resulted in significant overproduction of NO on postoperative day 1, which was significantly inhibited by pretreatment with GB. (D) No significant differences in NO levels were observed among the three groups on postoperative day 3. (E) Compared with the control group, surgery resulted in significant overproduction of MDA in the hippocampus on postoperative day 1, which was significantly inhibited by pretreatment with GB. (F) No significant differences in MDA levels were observed among the three groups on postoperative day 3 . Data are presented as the mean \pm SEM ( $n=6$ per group). ${ }^{*} \mathrm{P}<0.05$ vs. control; ${ }^{*} \mathrm{P}<0.05$ vs. surgery. GB, ginkgolide B; SOD, superoxide dismutase; NO, nitric oxide; MDA, malondialdehyde; Con, control; Sur, surgery.

those in the control group ( $\mathrm{P}<0.05$; Fig. $6 \mathrm{~A}$ and $\mathrm{B})$. By contrast, Bax expression levels in the surgery group were significantly increased compared with those in the control group $(\mathrm{P}<0.05$; Fig. 6A and C). Compared with the surgery group, pretreatment with GB significantly increased Bcl-2 expression levels on postoperative day 3, whereas GB pretreatment significantly attenuated surgery-induced Bax upregulation $(\mathrm{P}<0.05$; Fig. 6A-C). Furthermore, the ratio of Bax/Bcl-2, which indicates activation of the proapoptotic signaling pathway (26), was significantly higher in the surgery group compared with that in the control group $(\mathrm{P}<0.05$; Fig. 6D). Compared with the surgery group, GB pretreatment significantly decreased the Bax/Bcl-2 ratio $(\mathrm{P}<0.05$; Fig. $6 \mathrm{D})$. Thus, the results suggested that $\mathrm{GB}$ pretreatment inhibited surgery-induced apoptosis.

Hematoxylin and eosin staining was conducted to evaluate morphological alterations of neurons in the hippocampus on postoperative day 3 . The neurons were round and tightly arranged in the CA1 and CA3 regions in the control group
(Fig. 7A and D). However, compared with control group, the neurons in the CA1 and CA3 regions of the surgery group displayed greater shrinkage, a looser arrangement and darker staining (Fig. 7B and E). In addition, compared with the surgery group, markedly fewer dark-stained and paramorphic neurons were observed in the CA1 and CA3 regions in the surgery + GB group (Fig. 7C and F). Compared with that in the surgery group, a significantly higher number of normal neurons were observed in the hippocampus of the surgery $+\mathrm{GB}$ group $(\mathrm{P}<0.05$; Fig. $7 \mathrm{G}$ and $\mathrm{H})$. The results indicated that pretreatment with GB reduced abdominal surgery-induced neuronal histological abnormalities in the hippocampal region.

\section{Discussion}

The results of the present study indicated an association between iNOS-mediated overproduction of NO and abdominal surgery-induced impairments in learning and memory. 
A

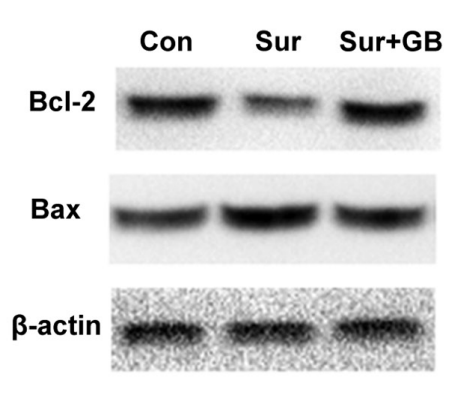

C

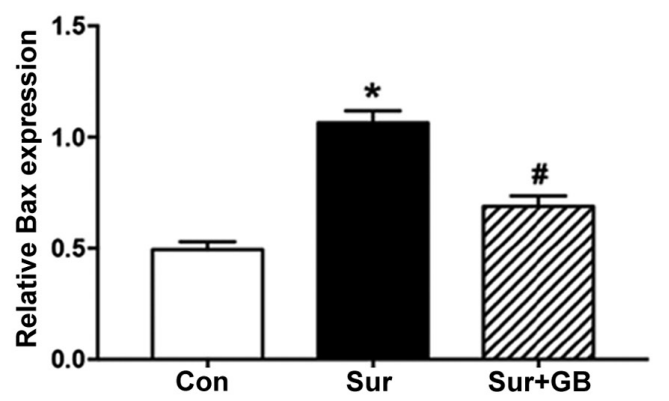

B

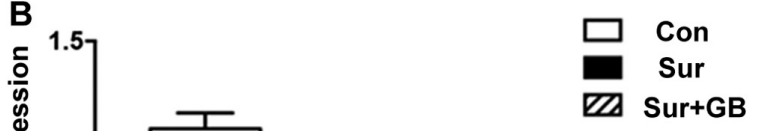

ZZ Sur+GB

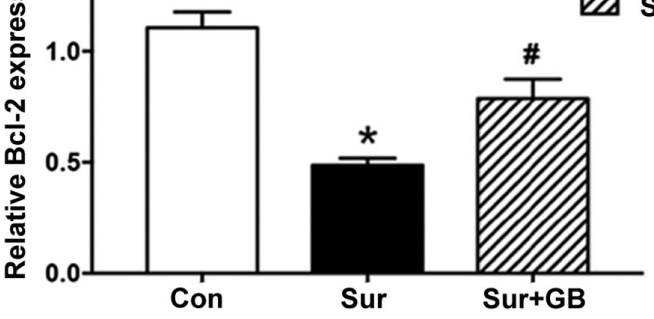

D

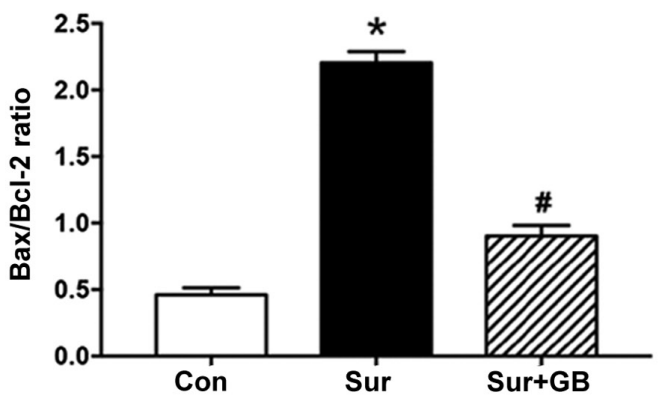

Figure 6. Effect of GB pretreatment on Bax and Bcl-2 expression levels in the hippocampus. Protein expression levels on postoperative day 3 were (A) determined via western blotting and semi-quantified for (B) Bcl-2, (C) Bax and (D) the Bax/Bcl-2 ratio. Data are presented as the mean \pm SEM ( $\mathrm{n}=4$ per group). ${ }^{*} \mathrm{P}<0.05$ vs. control; ${ }^{\#} \mathrm{P}<0.05$ vs. surgery. GB, ginkgolide $\mathrm{B}$; Con, control; Sur, surgery.
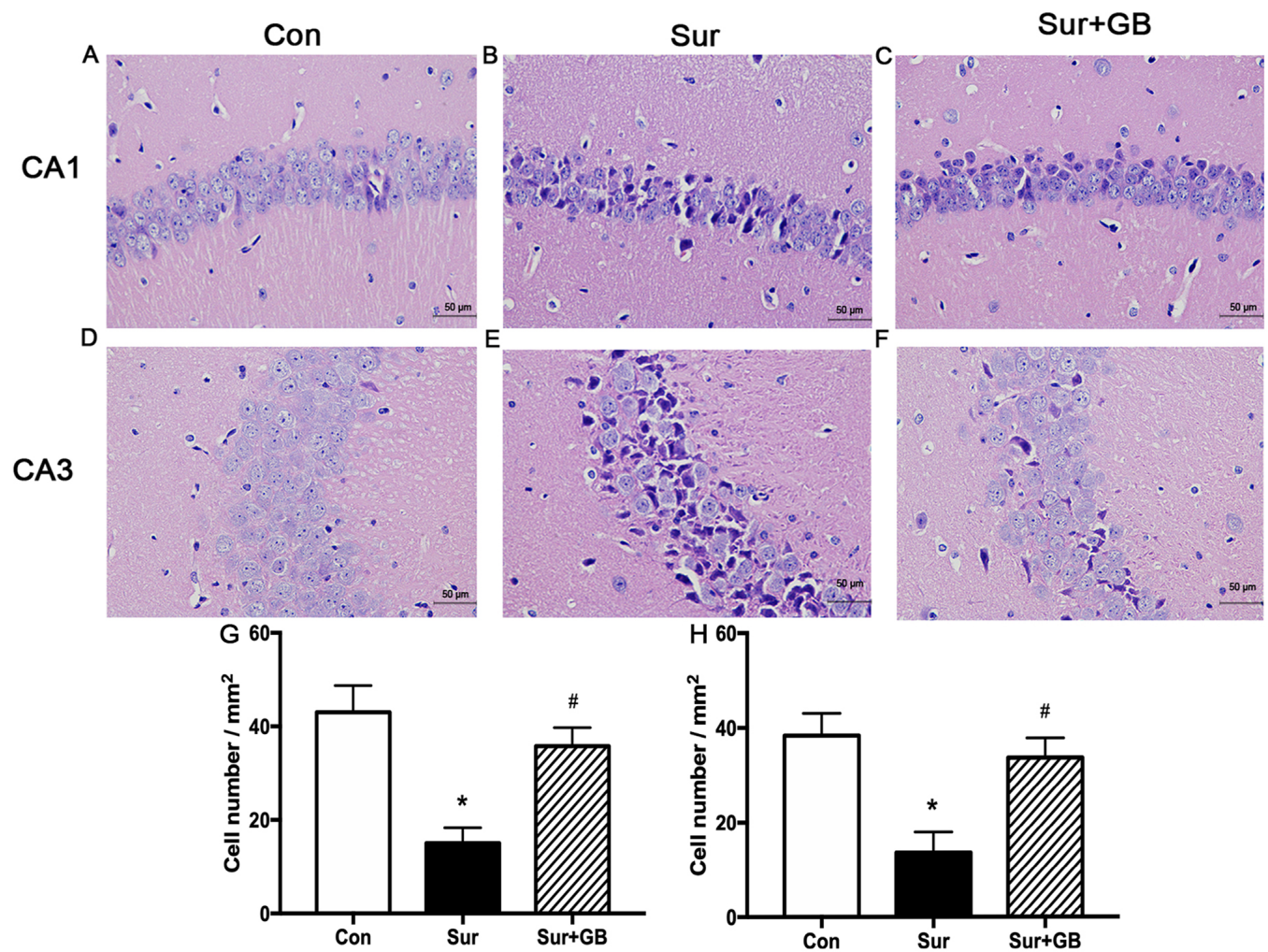

Figure 7. Hematoxylin and eosin staining of neuronal morphological alterations in the hippocampus. Representative images of the CA1 region of the hippocampus in (A) control, (B) surgery and (C) surgery + GB groups (magnification, x400). Representative images of the CA3 region of the hippocampus in (D) control, (E) surgery and (F) surgery + GB groups (magnification, $x 400$ ). Quantification of the number of normal neurons in the (G) CA1 and (H) CA3 regions of the hippocampus. Data are presented as the mean $\pm \mathrm{SEM}$ ( $\mathrm{n}=3$ per group). ${ }^{*} \mathrm{P}<0.05$ vs. control; ${ }^{\#} \mathrm{P}<0.05$ vs. surgery. GB, ginkgolide $\mathrm{B}$; Con, control; Sur, surgery.

Moreover, GB pretreatment improved cognitive dysfunction induced by abdominal surgery under general anesthesia. Therefore, the present study indicated that the protective role of GB against PND may be associated with its ability to reduce iNOS production and subsequent NO production, as well as its capacity to decrease neuronal loss and apoptosis. 
PND, as a common complication occurring after surgery, has attracted increasing attention from perioperative physicians. According to a previous study, a PND model was constructed via abdominal laparotomy in the present study (27). PND can present in young and middle-aged patients $(28,29)$. Therefore, adult mice were used to construct the PND model in the present study, according to a previous study (30). To determine the effects of $1400 \mathrm{~W}$ and GB on surgery-induced learning and memory impairment, the FCT, which has frequently been used in studies investigating PND, was performed to assess the cognitive function of mice in each group (24,31). An OFT was conducted to assess exploratory and locomotor activities (21). The behavioral studies demonstrated that surgery resulted in deficits in hippocampus-dependent learning and memory without influencing exploratory and locomotor activities. Consistent with the results reported by Sun et al (32), the present study demonstrated that hippocampus-dependent cognitive functions were vulnerable to surgery under anesthesia in young adult mice. Moreover, pretreatment with $1400 \mathrm{~W}$ or GB significantly improved surgery-induced cognitive dysfunction.

iNOS can be expressed in astrocytes, microglia, neurons and endothelial cells in the central nervous system under certain pathological conditions (33). The present study demonstrated higher expression of iNOS and levels of NO in the surgery group on postoperative day 1 compared with the control group. On postoperative day $1, \mathrm{NO}$ overproduction in the surgery group was significantly reduced by pretreatment with the specific iNOS inhibitor $1400 \mathrm{~W}$, which suggested that iNOS was involved in the process of PND via mediation of NO overproduction.

As one of the most important antioxidants in the central nervous system, SOD can protect cells from oxidative damage by promoting the transformation of superoxide anions to oxygen molecules and hydrogen peroxide (34). MDA is the final product of lipid peroxidation induced by ROS (35). In the present study, compared with the control group, the surgery group displayed significantly decreased SOD activity in the hippocampus and prefrontal cortex on postoperative day 1 and in the hippocampus on postoperative day 3. Higher MDA content was found in the hippocampus of the surgery group on postoperative day 1 . These changes indicated that anesthesia and surgery resulted in decreased antioxidants and increased ROS. The results of the present study were consistent with a previous study that reported a significant decrease in SOD activity in the $24 \mathrm{~h}$ following surgery (5). Subsequently, increased superoxide anion, a species of ROS, and NO induced by surgery could promote the production of $\mathrm{ONOO}^{-}$, which could lead to neuronal apoptosis and destruction $(36,37)$.

In the present study, compared with the control group, significant NO and MDA upregulation in the hippocampus in the surgery group was observed on postoperative day 1, but not on postoperative day 3. However, impaired cognitive results were observed on postoperative days 1 and 3. Several reasons could account for the aforementioned results. First, NO has been indicated to be an unstable gas with a short half-life. However, even in a very short period of time, large amounts of ONOO could be generated via the NO/superoxide anion pathway (38). Second, in addition to promoting lipid peroxidation, $\mathrm{ONOO}^{-}$could promote nitrosylation of membrane protein thiols in the mitochondria, mitochondrial damage, protein oxidation and DNA oxidation (39). As a result, other pathophysiological mechanisms mediated by $\mathrm{ONOO}^{-}$may underlie PND on postoperative day 3.

Numerous studies have indicated the protective role of GB in the context of neurodegenerative diseases. Gu et al (17) reported that GB could partially reverse iNOS upregulation in a transient middle cerebral artery occlusion mouse model. $\mathrm{NF}-\kappa \mathrm{B}$ is an important transcription factor in regulating iNOS expression (40). A previous study demonstrated that GB downregulated iNOS expression by inhibiting NF-kB activation (41). In the present study, surgery-induced iNOS upregulation and NO overproduction in the hippocampus on postoperative day 1 were significantly inhibited by pretreatment with GB. In addition, pretreatment with GB significantly decreased surgery-induced downregulation of SOD in the hippocampus and prefrontal cortex on postoperative day 1 , and in the hippocampus on postoperative day 3. Overproduction of MDA in the hippocampus on postoperative day 1 was also inhibited by pretreatment of GB. A previous study reported similar properties of GB. GB pretreatment protected SH-5YSY cells against A $\beta 1-42$ by reducing lipid peroxidation and restoring antioxidant activities (42). Furthermore, GB restored SOD activity and decreased MDA content in hypoxia-exposed rats (43). The present study demonstrated a significantly higher ratio of proapoptotic Bax/Bcl-2 in the surgery group compared with that in the control group, which was significantly inhibited by GB pretreatment. Surgery-induced neuronal histological abnormalities in the CA1 and CA3 regions were prevented by GB pretreatment. The results suggest that the therapeutic effects of GB on PND were associated with the inhibition of iNOS-mediated NO production, as well as the alleviation of neuronal apoptosis and destruction, which were possibly induced by the elevation of $\mathrm{ONOO}^{-}$. Collectively, the results of the present study may provide novel insight for the treatment of PND.

However, the present study had several limitations. First, an inhibitor of iNOS, $1400 \mathrm{~W}$, was used to investigate the role of iNOS in PND. However, employing iNOS gene knockout mice would provide more powerful evidence. Second, without a GB pretreatment and iNOS overexpression group, it cannot be concluded from the results of the present study that iNOS mediated the protective effect of GB on PND. In addition, testing relevant indicators at different time points would aid in identifying the dynamic alterations of experimental indexes post-surgery. However, to reduce the number of animals used in the present study, relevant indicators were assessed on postoperative days 1 and 3 , according to previous studies $(30,44)$. Therefore, the regulatory mechanisms underlying the effects of GB on iNOS during the process of PND require further investigation.

In conclusion, the present study indicated that surgery may cause PND by promoting elevations in iNOS/NO and ROS, downregulating SOD and inducing neuronal damage. GB treatment may serve a protective role in PND by inhibiting iNOS upregulation and NO overproduction, inhibiting SOD downregulation and alleviating neuronal damage. The results suggested that iNOS may serve as a potential therapeutic target 
for PND, and GB may serve as a novel drug for the prevention of PND. Future studies should focus on further understanding the mechanisms underlying iNOS and NO in PND to aid with the discovery of multiple therapeutic targets.

\section{Acknowledgements}

Not applicable.

\section{Funding}

The present study was supported by the National Natural Science Foundation of China (grant nos. 81541117 and 81371199).

\section{Availability of data and materials}

The datasets used and/or analyzed during the current study are available from the corresponding author on reasonable request.

\section{Authors' contributions}

AW and PH designed the present study. YH and RS analyzed the data. TL and DL performed the experiments. TL was a major contributor in writing the manuscript. DL and $\mathrm{PH}$ revised the manuscript. CW and WS contributed new reagents, and performed the data collection and interpretation. All authors confirm the authenticity of all the raw data, and read and approved the final manuscript.

\section{Ethics approval and consent to participate}

All experiments were approved by the Animal Experiments and Experimental Animal Welfare Committee of Capital Medical University and performed under the regulations of the Medical Research Center of Beijing Chao-Yang Hospital.

\section{Patient consent for publication}

Not applicable.

\section{Competing interests}

The authors declare that they have no competing interests.

\section{References}

1. Evered L, Silbert B, Knopman DS, Scott DA, DeKosky ST, Rasmussen LS, Oh ES, Crosby G, Berger M and Eckenhoff RG; Nomenclature Consensus Working Group: Recommendations for the nomenclature of cognitive change associated with anaesthesia and surgery-2018. Anesthesiology 129: 872-879, 2018.

2. Huang S, Hu H, Cai YH and Hua F: Effect of parecoxib in the treatment of postoperative cognitive dysfunction: A systematic review and meta-analysis. Medicine (Baltimore) 98: e13812, 2019.

3. Androsova G, Krause R, Winterer G and Schneider R: Biomarkers of postoperative delirium and cognitive dysfunction. Front Aging Neurosci 7: 112, 2015.

4. Netto MB, de Oliveira Junior AN, Goldim M, Mathias K, Fileti ME, da Rosa N, Laurentino AO, de Farias BX, Costa AB, Rezin GT, et al: Oxidative stress and mitochondrial dysfunction contributes to postoperative cognitive dysfunction in elderly rats. Brain Behav Immun 73: 661-669, 2018.
5. Zhang J, Gao J, Guo G, Li S, Zhan G, Xie Z, Yang C and Luo A: Anesthesia and surgery induce delirium-like behavior in susceptible mice: The role of oxidative stress. Am J Transl Res 10: 2435-2444, 2018

6. Dröge W: Free radicals in the physiological control of cell function. Physiol Rev 82: 47-95, 2002.

7. Venturelli M, Pedrinolla A, Boscolo Galazzo I, Fonte C, Smania N, Tamburin S, Muti E, Crispoltoni L, Stabile A, Pistilli A, et al: Impact of nitric oxide bioavailability on the progressive cerebral and peripheral circulatory impairments during aging and Alzheimer's disease. Front Physiol 9: 169, 2018.

8. Stephan BCM, Harrison SL, Keage HAD, Babateen A, Robinson L and Siervo M: Cardiovascular disease, the nitric oxide pathway and risk of cognitive impairment and dementia. Curr Cardiol Rep 19: 87, 2017.

9. Tse JKY: Gut microbiota, nitric oxide, and microglia as prerequisites for neurodegenerative disorders. ACS Chem Neurosci 8: 1438-1447, 2017.

10. Takahashi M, Chin Y, Nonaka T, Hasegawa M, Watanabe N and Arai T: Prolonged nitric oxide treatment induces tau aggregation in SH-SY5Y cells. Neurosci Lett 510: 48-52, 2012.

11. Chinta SJ and Andersen JK: Nitrosylation and nitration of mitochondrial complex I in Parkinson's disease. Free Radic Res 45: 53-58, 2011

12. Asiimwe N, Yeo SG, Kim MS, Jung J and Jeong NY: Nitric oxide: Exploring the contextual link with Alzheimer's disease. Oxid Med Cell Longev 2016: 7205747, 2016.

13. Zhang Y, Xiong S, Liu P, Liu W, Wang Q, Liu Y, Tan H, Chen X, Shi X, Wang Q and Chen T: Polymeric nanoparticles-based brain delivery with improved therapeutic efficacy of ginkgolide B in Parkinson's disease. Int J Nanomedicine 15: 10453-10467, 2020.

14. Sharma HS, Drieu K, Alm P and Westman J: Role of nitric oxide in blood-brain barrier permeability, brain edema and cell damage following hyperthermic brain injury. An experimental study using EGB-761 and Gingkolide B pretreatment in the rat. Acta Neurochir Suppl 76: 81-86, 2000.

15. Liu Q, Jin Z, Xu Z, Yang H, Li L, Li G, Li F, Gu S, Zong S, Zhou J, et al: Antioxidant effects of ginkgolides and bilobalide against cerebral ischemia injury by activating the Akt/Nrf2 pathway in vitro and in vivo. Cell Stress Chaperones 24: 441-452, 2019.

16. Deng Y, Fang W, Li Y, Cen J, Fang F, Lv P, Gong S and Mao L: Blood-brain barrier breakdown by PAF and protection by XQ-1H due to antagonism of PAF effects. Eur J Pharmacol 616: 43-47, 2009.

17. Gu JH, Ge JB, Li M, Wu F, Zhang W and Qin ZH: Inhibition of NF- $\mathrm{KB}$ activation is associated with anti-inflammatory and anti-apoptotic effects of ginkgolide B in a mouse model of cerebral ischemia/reperfusion injury. Eur J Pharm Sci 47: 652-660, 2012.

18. Fang W, Sha L, Kodithuwakku ND, Wei J, Zhang R, Han D, Mao L andf Li Y: Attenuated blood-brain barrier dysfunction by XQ-1H following ischemic stroke in hyperlipidemic rats. Mol Neurobiol 52: 162-175, 2015.

19. Rosczyk HA, Sparkman NL and Johnson RW: Neuroinflammation and cognitive function in aged mice following minor surgery. Exp Gerontol 43: 840-846, 2008.

20. Tian XS, Tong YW, Li ZQ, Li LX, Zhang T, Ren TY, Zhou T, Wang HC, Zhan R, Sun Y, et al: Surgical stress induces brain-derived neurotrophic factor reduction and postoperative cognitive dysfunction via glucocorticoid receptor phosphorylation in aged mice. CNS Neurosci Ther 21: 398-409, 2015.

21. Seibenhener ML and Wooten MC: Use of the open field maze to measure locomotor and anxiety-like behavior in mice. $\mathrm{J}$ Vis Exp 6: e52434, 2015.

22. Zhang Z, Li X, Li F and An L: Berberine alleviates postoperative cognitive dysfunction by suppressing neuroinflammation in aged mice. Int Immunopharmacol 38: 426-433, 2016.

23. Saxe MD, BattagliaF, Wang JW, Malleret G, DavidDJ, Monckton JE, Garcia AD, Sofroniew MV, Kandel ER, Santarelli L, et al: Ablation of hippocampal neurogenesis impairs contextual fear conditioning and synaptic plasticity in the dentate gyrus. Proc Natl Acad Sci USA 103: 17501-17506, 2006.

24. Xu Z, Dong Y, Wang H, Culley DJ, Marcantonio ER, Crosby G, Tanzi RE, Zhang Y and Xie Z: Age-dependent postoperative cognitive impairment and Alzheimer-related neuropathology in mice. Sci Rep 4: 3766, 2014.

25. Winocur G, Wojtowicz JM, Sekeres M, Snyder JS and Wang S: Inhibition of neurogenesis interferes with hippocampusdependent memory function. Hippocampus 16: 296-304, 2006.

26. Korsmeyer SJ, Shutter JR, Veis DJ, Merry DE and Oltvai ZN: Bcl-2/Bax: A rheostat that regulates an anti-oxidant pathway and cell death. Semin Cancer Biol 4: 327-332, 1993. 
27. Hovens IB, van Leeuwen BL, Mariani MA, Kraneveld AD and Schoemaker RG: Postoperative cognitive dysfunction and neuroinflammation; cardiac surgery and abdominal surgery are not the same. Brain Behav Immun 54: 178-193, 2016.

28. Monk TG, Weldon BC, Garvan CW, Dede DE, van der Aa MT, Heilman KM and Gravenstein JS: Predictors of cognitive dysfunction after major noncardiac surgery. Anesthesiology 108: $18-30,2008$.

29. Shu AH, Wang Q and Chen XB: Effect of different depths of anesthesia on postoperative cognitive function in laparoscopic patients: A randomized clinical trial. Curr Med Res Opin 31: 1883-1887, 2015

30. Xiong C, Liu J, Lin D, Zhang J, Terrando $\mathrm{N}$ and $\mathrm{Wu} \mathrm{A}$ Complement activation contributes to perioperative neurocognitive disorders in mice. J Neuroinflammation 15: 254, 2018.

31. Zhang J, Zhu S, Jin P, Huang Y, Dai Q, Zhu Q, Wei P, Yang Z, Zhang L, Liu H, et al: Graphene oxide improves postoperative cognitive dysfunction by maximally alleviating amyloid beta burden in mice. Theranostics 10: 11908-11920, 2020.

32. Sun L, Dong R, Xu X, Yang X and Peng M: Activation of cannabinoid receptor type 2 attenuates surgery-induced cognitive impairment in mice through anti-inflammatory activity. J Neuroinflammation 14: 138, 2017.

33. Zhou XY, Zhang F, Ying CJ, Chen J, Chen L, Dong J, Shi Y, Tang M, Hu XT, Pan ZH, et al: Inhibition of iNOS alleviates cognitive deficits and depression in diabetic mice through downregulating the $\mathrm{NO} / \mathrm{sGC} / \mathrm{cGMP} / \mathrm{PKG}$ signal pathway. Behav Brain Res 322: 70-82, 2017.

34. Clausen A, Doctrow S and Baudry M: Prevention of cognitive deficits and brain oxidative stress with superoxide dismutase/catalase mimetics in aged mice. Neurobiol Aging 31: $425-433,2010$

35. Greilberger J, Koidl C, Greilberger M, Lamprecht M, Schroecksnadel K, Leblhuber F, Fuchs D and Oettl K: Malondialdehyde, carbonyl proteins and albumin-disulphide as useful oxidative markers in mild cognitive impairment and Alzheimer's disease. Free Radic Res 42: 633-638, 2008

36. Shi Q, Liu X, Wang N, Zheng X, Ran J, Liu Z, Fu J and Zheng J: 1400W ameliorates acute hypobaric hypoxia/reoxygenation-induced cognitive deficits by suppressing the induction of inducible nitric oxide synthase in rat cerebral cortex microglia. Behav Brain Res 319: 188-199, 2017.
37. Moldogazieva NT, Mokhosoev IM, Feldman NB and Lutsenko SV: ROS and RNS signalling: Adaptive redox switches through oxidative/nitrosative protein modifications. Free Radic Res 52: 507-543, 2018.

38. Chitnis T and Weiner HL: CNS inflammation and neurodegeneration. J Clin Invest 127: 3577-3587, 2017.

39. Hemanth Kumar K, Tamatam A, Pal A and Khanum F: Neuroprotective effects of cyperus rotundus on SIN-1 induced nitric oxide generation and protein nitration: Ameliorative effect against apoptosis mediated neuronal cell damage. Neurotoxicology 34 150-159, 2013.

40. Ranjan R, Karpurapu M, Rani A, Chishti AH and Christman JW: Hemozoin regulates iNOS expression by modulating the transcription factor NF- $\kappa \mathrm{B}$ in macrophages. Biochem Mol Biol J 2: $10,2016$.

41. Woo CW, Cheung F, Chan VW, Siow YL and O K: Homocysteine stimulates inducible nitric oxide synthase expression in macrophages: Antagonizing effect of ginkgolides and bilobalide. Mol Cell Biochem 243: 37-47, 2003

42. Gill I, Kaur S, Kaur N, Dhiman M and Mantha AK: Phytochemical ginkgolide B attenuates amyloid- $\beta 1-42$ induced oxidative damage and altered cellular responses in human neuroblastoma SH-SY5Y Cells. J Alzheimers Dis 60 (Suppl 1) S25-S40, 2017.

43. Li W, Qinghai S, Kai L, Xue M, Lili N, Jihua R, Zhengxiang L, Xiaoling L, Di G, Qi Y, et al: Oral administration of ginkgolide B alleviates hypoxia-induced neuronal damage in rat hippocampus by inhibiting oxidative stress and apoptosis. Iran J Basic Med Sci 22: 140-145, 2019.

44. Qian XL, Zhang W, Liu MZ, Zhou YB, Zhang JM, Han L, Peng YM, Jiang JH and Wang QD: Dexmedetomidine improves early postoperative cognitive dysfunction in aged mice. Eur J Pharmacol 746: 206-212, 2015. International (CC BY-NC-ND 4.0) License. 